OPEN ACCESS

Edited by:

Vance L. Trudeau, University of Ottawa, Canada

Reviewed by:

Vincent M. Cassone,

University of Kentucky, United States Maria Claudia Gonzalez Deniselle, CONICET Instituto de Biología y Medicina Experimental (IBYME), Argentina

*Correspondence: Shogo Haraguchi shogo.haraguchi@gmail.com

Specialty section: This article was submitted to Neuroendocrine Science, a section of the journal Frontiers in Endocrinology

Received: 30 April 2020

Accepted: 06 July 2020

Published: 11 August 2020

Citation

Haraguchi S and Tsutsui K (2020) Pineal Neurosteroids: Biosynthesis and Physiological Functions.

Front. Endocrinol. 11:549 doi: 10.3389/fendo.2020.00549

\section{Pineal Neurosteroids: Biosynthesis and Physiological Functions}

\author{
Shogo Haraguchi ${ }^{1 *}$ and Kazuyoshi Tsutsui ${ }^{2}$ \\ ${ }^{1}$ Department of Biochemistry, Showa University School of Medicine, Tokyo, Japan, ${ }^{2}$ Graduate School of Integrated Sciences \\ for Life, Hiroshima University, Hiroshima, Japan
}

Similar to the adrenal glands, gonads, and placenta, vertebrate brains also produce various steroids, which are known as "neurosteroids." Neurosteroids are mainly synthesized in the hippocampus, hypothalamus, and cerebellum; however, it has recently been discovered that in birds, the pineal gland, a photosensitive region in the brain, produces more neurosteroids than other brain regions. A series of experiments using molecular and biochemical techniques have found that the pineal gland produces various neurosteroids, including sex steroids, de novo from cholesterol. For instance, allopregnanolone and $7 \alpha$-hydroxypregnenolone are actively produced in the pineal gland, unlike in other brain regions. Pineal $7 \alpha$-hydroxypregnenolone, an up-regulator of locomotion, enhances locomotor activity in response to light stimuli in birds. Additionally, pineal allopregnanolone acts on Purkinje cells in the cerebellum and prevents neuronal apoptosis within the developing cerebellum in juvenile birds. Furthermore, exposure to light during nighttime hours can cause loss of diurnal variations of pineal allopregnanolone synthesis during early posthatch life, eventually leading to cerebellar Purkinje cell death in juvenile birds. In light of these new findings, this review summarizes the biosynthesis and physiological functions of pineal neurosteroids. Given that the circadian rhythms of individuals in modern societies are constantly interrupted by artificial light exposure, these findings in birds, which are excellent model diurnal animals, may have direct implications for addressing problems regarding the mental health and brain development of humans.

Keywords: allopregnanolone, $7 \alpha$-hydroxypregnenolone, neurosteroid, pineal gland, cerebellum, light

\section{INTRODUCTION}

Similar to the gonads and placenta, vertebrate brains actively also produce various steroid hormones. These steroid hormones produced in the brain are named "neurosteroids." The production of neurosteroids was demonstrated firstly in mammals, and then in other vertebrates $(1-5)$. Thus, neurosteroid production appears to be a universal feature of the brain in vertebrates.

It is known that neurosteroids are produced in glial cells and neurons of the central and peripheral nervous systems $(1,5)$. However, we have demonstrated that the pineal gland produces neurosteroids from cholesterol in birds during early posthatch period (6-8). Notably, allopregnanolone (also known as $3 \alpha, 5 \alpha$-tetrahydroprogesterone; $3 \alpha, 5 \alpha$-THP) and $7 \alpha-$ hydroxypregnenolone are the two major neurosteroids produced in the pineal gland $(6,7)$. Of these two, pineal allopregnanolone prevents the death of developing Purkinje cells $(7,8)$, and pineal $7 \alpha-$ hydroxypregnenolone functions as an up-regulator of locomotion, regulating locomotor activity in response to light stimuli in birds (6). 


\section{BIOSYNTHESIS OF PINEAL NEUROSTEROIDS}

The pineal glands of vertebrates respond to light stimuli and fulfill important functions in the organization of circadian rhythms. The secretion of melatonin, a major hormone produced by the pineal gland, shows a clear daily rhythm with its peak concentration occurring at night $(7,9)$. However, it was not known whether the pineal gland produces neurosteroids until recently. We have recently demonstrated that the pineal gland is a newly found neurosteroidogenic organ producing a variety of neurosteroids from cholesterol $(6,7)$ (Figure 1).

Pregnenolone is an anabolic intermediate of most endogenous steroid hormones and is produced from cholesterol through the mitochondrial cholesterol side chain cleavage enzyme cytochrome P450scc (P450scc; encoded by the Cyp11a gene). We have demonstrated by transcription-polymerase chain reaction (RT-PCR) that the pineal gland in juvenile birds expresses P450scc mRNA $(6,7)$ (Figure 1). The protein product of this mRNA is localized in the cells that form the follicular structures in the pineal glands of birds (7). We have demonstrated by high-performance liquid chromatography (HPLC) with radioactive flow detector analysis that ${ }^{3} \mathrm{H}$ cholesterol is converted to radioactive pregnenolone when incubated with pineal gland extract from juvenile birds $(6,7)$. This observation has confirmed the presence of functional P450scc in the pineal gland (Figure 1), which has also been detected by gas chromatography-mass spectrometry (GC/MS) (7). Subsequent RT-PCR-based assessment has revealed that key steroidogenic enzymes, cytochrome P450 $7 \alpha$-hydroxylase (P4507 $\alpha$; encoded by the Cyp7b gene), $3 \alpha$-hydroxysteroid dehydrogenase $/ \Delta^{5}-\Delta^{4}$-isomerase ( $3 \alpha$-HSD; encoded by the $H s d 3 a$ gene), $3 \beta$-hydroxysteroid dehydrogenase $/ \Delta^{5}-\Delta^{4}$ -

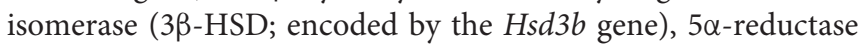
(encoded by the $S r d 5 a$ gene), $5 \beta$-reductase (encoded by the Srd5b gene), cytochrome P450 17 $\alpha$-hydroxylase/c17,20-lyase (P45017 $\alpha$,lyase; encoded by the Cyp17 gene), 17 $\beta$-hydroxysteroid dehydrogenase (17 $\beta$-HSD; encoded by the Hsd17b gene), and cytochrome P450 aromatase (P450arom; encoded by the Cyp 19 gene), are expressed in the pineal gland of birds $(6,7)$ (Figure 1).

We further demonstrated that steroid hormones are indeed present in the pineal gland. Incubation of ${ }^{3} \mathrm{H}$-pregnenolone with pineal glands from posthatch birds generates $7 \alpha$ - and/or $7 \beta$-hydroxypregnenolone by the action of $\mathrm{P} 4507 \alpha$ found in the pineal glands (7) (Figure 1). In addition to these neurosteroid isomers, progesterone, allopregnanolone ( $3 \alpha, 5 \alpha-$ THP) and/or epipregnanolone ( $3 \beta, 5 \beta$-THP), androstenedione, testosterone, $5 \alpha-$ and/or $5 \beta$-dihydrotestosterone, and estradiol-17 $\beta$ are also produced (7) (Figure 1). These ex vivo observations have confirmed that the pineal glands in juvenile birds have the biosynthetic machinery for major steroid hormones, which have also been verified to be produced as neurosteroids in vivo (7) (Figure 1). Although HPLC analysis has failed to resolve the isomers of these hormones, such as $7 \alpha$-/ $\beta$-hydroxypregnenolone, allo/epipregnanolone, and $5 \alpha$-/ $\beta$-dihydrotestosterone, several sets of isomers have been successfully isolated by GC/MS analysis (7). Especially,
$7 \alpha$-hydroxypregnenolone and allopregnanolone are actively released $(6,7)$.

Taken together, these findings indicate that the pineal gland in juvenile birds produces various neurosteroids from cholesterol. Accordingly, this is the first demonstration of neurosteroid synthesis in the pineal gland in a vertebrate.

\section{PHYSIOLOGICAL FUNCTION OF PINEAL $7 \alpha$-HYDROXYPREGNENOLONE IN LIGHT-DEPENDENT LOCOMOTION}

The chick pineal gland is used as a model for studies on the light-dependent phase-shifting mechanism of the circadian clock (10). To search for genes involved in this mechanism, a differential GeneChip analysis has been performed. This transcriptomics analysis has identified the light-induced transcriptional activation of the full set of genes in the pineal gland involved in cholesterol biosynthesis (6). When the pineal gland was exposed to light, it produced cholesterol and $7 \alpha$-hydroxypregnenolone ex vivo. Interestingly, this light-induced production of $7 \alpha-$ hydroxypregnenolone occurred only when the gland was exposed to light at early night but not at late night or during the daytime. During early night time, the circadian clock is sensitive to light, which causes phase-delay of the clock (10). Thus, the light-sensitive pineal production of $7 \alpha$-hydroxypregnenolone appears to be regulated by the circadian clock.

In vertebrates, an intracerebroventricular injection of $7 \alpha$-hydroxypregnenolone activates locomotor activities (11-15). Thus, the intracerebroventricular injection of $7 \alpha-$ hydroxypregnenolone was administered in a dose-dependent manner at early night in chicks (6). After the injection, chicks were placed individually for locomotor activity measurement in an open field apparatus for $20 \mathrm{~min}$. Spontaneous locomotor activities of chicks were stimulated by the intracerebroventricular injection of $7 \alpha$-hydroxypregnenolone in a dose-dependent manner (6). Furthermore, when chicks are exposed to light during early night time, their locomotor activities reach the daytime level (6). These results suggest that pineal $7 \alpha-$ hydroxypregnenolone reaches the target sites within the brain by volume transmission (16) upon light exposure at early night.

\section{PHYSIOLOGICAL FUNCTION OF PINEAL ALLOPREGNANOLONE IN PURKINJE CELL SURVIVAL DURING DEVELOPMENT}

$7 \alpha$-Hydroxypregnenolone and allopregnanolone are actively released during early posthatch period compared with adulthood (7). Therefore, $7 \alpha$-hydroxypregnenolone and allopregnanolone may play key roles in birds during early posthatch period. In vertebrates, pinealectomy decreases cell number in the developing brain $(17,18)$. These findings suggest that these major neurosteroids secreted from the pineal gland are involved in the development of brain cells. 


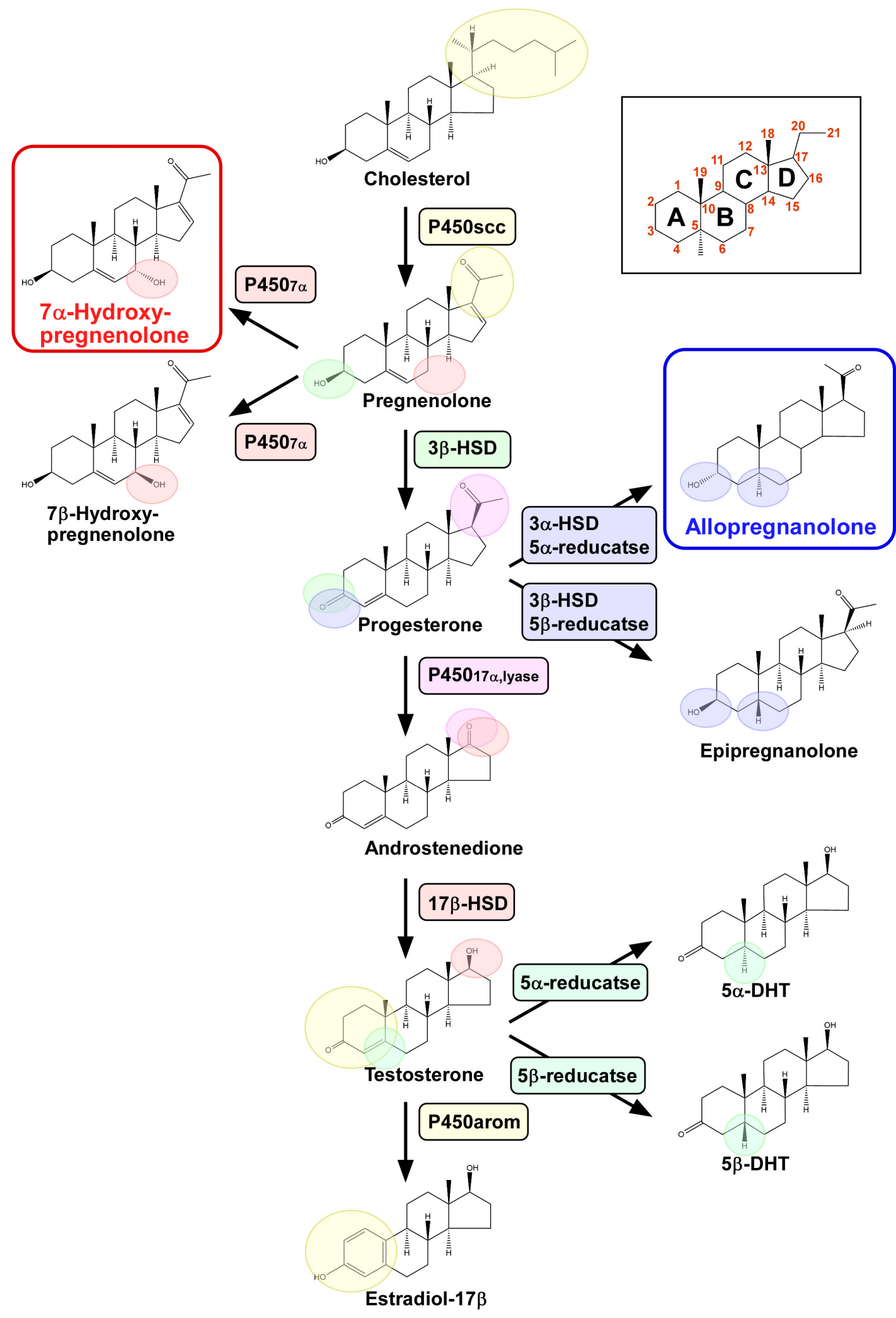

FIGURE 1 | Biosynthetic pathways of pineal neurosteroids. Allopregnanolone and $7 \alpha$-hydroxypregnenolone are the major neurosteroids produced in the pineal gland

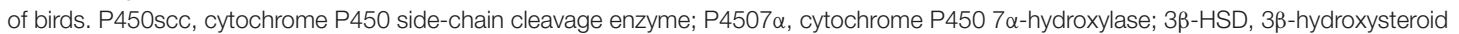

dehydrogenase $/ \Delta^{5}$ - $\Delta^{4}$-isomerase; $3 \alpha$-HSD, $3 \alpha$-hydroxysteroid dehydrogenase $/ \Delta^{5}$ - $\Delta^{4}$-isomerase; $5 \alpha$-reductase; $5 \beta$-reductase; P45017 $\alpha$,lyase, cytochrome P450

$17 \alpha$-hydroxylase/c17,20-lyase; $17 \beta$-HSD, 17 $\beta$-hydroxysteroid dehydrogenase; and P450arom, cytochrome P450 aromatase. 
In chicks, pinealectomy decreases the concentration of allopregnanolone and the number of cerebellar Purkinje cells, whereas the supplementation of allopregnanolone to pinealectomized birds increases the concentration of allopregnanolone and recovers the number of Purkinje cells (7). Thus, pineal allopregnanolone is considered to be an essential factor for the normal development of cerebellar Purkinje cells. It thus appears that pineal allopregnanolone functions as an essential factor for Purkinje cells during posthatch period.

In addition, pinealectomy in juvenile birds increases the expression of active caspase- 3 in Purkinje cells, whereas allopregnanolone supplementation decreases the expression of active caspase-3 during posthatch period (7). Thus, the neuroprotective action of pineal allopregnanolone on cerebellar Purkinje cells is exerted by suppressing the activation of caspase- 3

(Figure 2).

Allopregnanolone acts mainly as a ligand of the $\gamma$ aminobutyric acid type $\mathrm{A}\left(\mathrm{GABA}_{\mathrm{A}}\right)$ receptor and may also act as an agonist of the membrane progesterone receptors $\alpha$ $(\mathrm{mPR} \alpha)$, as well as the $\operatorname{mPR} \beta$ and $\operatorname{mPR} \gamma(19-21)$. Therefore, either mPR siRNA or isoallopregnanolone, an antagonist of allopregnanolone, was delivered into the cerebellum of posthatched chicks. It was found that the silencing of $\mathrm{mPR} \alpha$ increases the number of Purkinje cells that express active caspase3 in the cerebellum of chicks (8). Furthermore, to uncover the mechanism of neuroprotective action of allopregnanolone
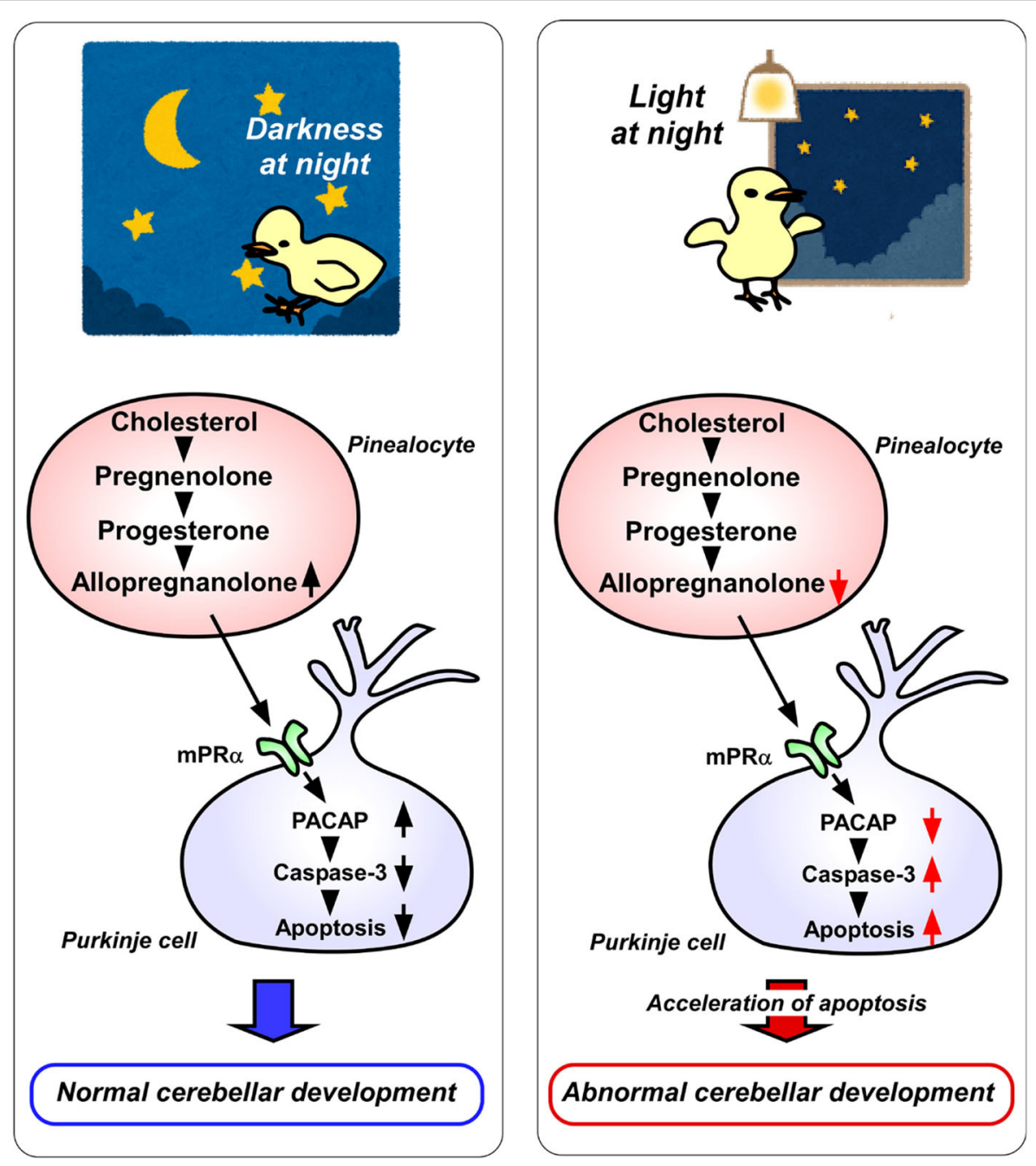

FIGURE 2 | A schematic model of the effect of pineal allopregnanolone on Purkinje cell survival immediately after hatching under a $12 / 12 \mathrm{~h}$ light/dark cycle or with $1 \mathrm{~h}$ light exposure during the dark period (light-at-night condition). (Left) panel The normal cerebellar development under a 12/12 h light/dark cycle during the first week after hatching. Pineal allopregnanolone induces the expression of pituitary adenylate cyclase-activating polypeptide (PACAP), a neuroprotective factor, through the membrane progestin receptor $\alpha(\mathrm{mPR} \alpha)$ receptor binding mechanism in Purkinje cells. Subsequently, PACAP inhibits the activation of caspase-3 that facilitates the apoptosis of cerebellar Purkinje cells. (Right) panel The abnormal cerebellar development under the light-at-night condition during the first week after hatching. The light-at-night condition disrupts the diurnal rhythm in pineal allopregnanolone synthesis. Decreased pineal allopregnanolone synthesis leads to decreased expression of PACAP in Purkinje cells. Consequently, the active caspase-3 level increases, inducing the apoptosis of Purkinje cells in the cerebellum. 
in cerebellar Purkinje cells, allopregnanolone action on the expression of neuroprotective/neurotoxic factors (22-26) has been investigated. Pinealectomy decreases the mRNA levels of pituitary adenylate cyclase-activating polypeptide (PACAP), a neuroprotective factor, in the cerebellum of juvenile birds (8). It has been found that a daily injection of allopregnanolone in pinealectomized juvenile birds upregulates PACAP relative to the levels in control birds (8). These findings show that PACAP mediates the neuroprotective action of pineal allopregnanolone through $\mathrm{mPR} \alpha$ receptor binding during cerebellar development (Figure 2).

\section{LIGHT-AT-NIGHT AFFECTS THE DEVELOPMENT OF CEREBELLUM THROUGH A MECHANISM MEDIATED BY PINEAL ALLOPREGNANOLONE ACTION}

It is known that environmental stimuli affect the development of animals including humans. In vertebrate brain development, a natural light-dark cycle promotes better brain development than constant conditions, such as constant light or constant darkness (27-31). However, the molecular mechanisms that control how environmental light conditions affect brain development remain unclear. The pineal gland is a photosensitive organ. To investigate whether light conditions are involved in the synthesis of allopregnanolone in the pineal gland, the birds have been incubated under either a $12 / 12 \mathrm{~h}$ light/dark (LD) cycle or LD cycle with $1 \mathrm{~h}$ light exposure during the dark period (light-at-night). Consequently, it has been found that the allopregnanolone concentration and synthesis during the dark period are higher in the pineal glands of LD birds than in those of light-at-night birds (8) (Figure 2). Furthermore, the number of cerebellar Purkinje cells is decreased by the light-at-night condition (8) (Figure 2). It is therefore considered that pineal allopregnanolone is a critical metabolite that affects cerebellar development in vertebrates, depending on the environmental light conditions.

\section{REFERENCES}

1. Baulieu EE. Neurosteroids: of the nervous system, by the nervous system, for the nervous system. Rec Prog Hormone Res. (1997) 52:1-32.

2. Tsutsui K, Ukena K, Takase M, Kohchi C, Lea. RW. Neurosteroid biosynthesis in vertebrate brains. Comp Biochem Physiol C. (1999) 124:1219. doi: 10.1016/S0742-8413(99)00065-1

3. Compagnone NA, Mellon SH. Neurosteroids: biosynthesis and function of these novel neuromodulators. Front Neuroendocrinol. (2000) 21:156. doi: 10.1006/frne.1999.0188

4. Tsutsui K, Matsunaga M, Miyabara H, Ukena K. Neurosteroid biosynthesis in the quail brain. J Exp Zool. (2006) 305A:733-42. doi: 10.1002/jez.a.302

5. Do-Rego JL, Seong JY, Burel D, Leprince J, Luu-The V, Tsutsui K, et al. Neurosteroid biosynthesis: enzymatic pathways and neuroendocrine regulation by neurotransmitters and neuropeptides. Front Neuroendocrinol. (2009) 30:259-301. doi: 10.1016/j.yfrne.2009.05.006

6. Hatori M, Hirota T, Iitsuka M, Kurabayashi N, Haraguchi S, Kokame $\mathrm{K}$, et al. Light-dependent and circadian clock-regulated activation of

\section{CONCLUSIONS}

This review summarized the recent data on pineal neurosteroids. Studies have indicated that the pineal gland produces neurosteroids from cholesterol in birds. Pineal $7 \alpha-$ hydroxypregnenolone regulates locomotion in response to light stimuli in birds. Pineal allopregnanolone prevents the death of developing Purkinje cells by suppressing neuronal apoptosis during development. In addition, circadian disruption by light exposure during nighttime leads to cell death of developing Purkinje cells through pineal allopregnanolone-dependent mechanisms in juvenile birds. These observations suggest that nighttime artificial light exposure in modern societies may also perturb the development of the human brain.

Almost all animals have circadian rhythms. However, modern life conditions chronically disrupt circadian rhythm through artificial light exposure. The disruption of circadian rhythm is associated with a decline in mental and physical health (32-34). The most potent circadian rhythm disruption is inappropriately timed bright light exposure (e.g., light-at-night). To investigate the effects of chronic circadian disruption in modern societies on mental and physical health, which is efficiently modeled by the light-at-night condition presented here, many studies have been conducted on mice. However, it is important for us to bear in mind that laboratory mice are mainly nocturnal animals, whereas humans are diurnal. Thus, birds are excellent animal models to uncover the effect of light-at-night on diurnal animals, including humans.

\section{AUTHOR CONTRIBUTIONS}

$\mathrm{SH}$ and KT wrote the manuscript. All authors contributed to the article and approved the submitted version.

\section{FUNDING}

This work was supported by JSPS Grants-in-Aid for Scientific Research (KAKENHI) Grant Numbers JP15K18571 and JP19K09033. sterol regulatory element-binding protein, X-box-binding protein 1, and heat shock factor pathways. Proc Natl Acad Sci USA. (2011) 108:48649. doi: 10.1073/pnas.1015959108

7. Haraguchi, S Hara, S Ubuka T, Mita M, Tsutsui K. Possible role of pineal allopregnanolone in Purkinje cell survival. Proc Natl Acad Sci USA. (2012) 109:21110-5. doi: 10.1073/pnas.1210804109

8. Haraguchi S, Kamata M, Tokita T, Tashiro KI, Sato M, Nozaki M, et al. Light-at-night exposure affects brain development through pineal allopregnanolone-dependent mechanisms. eLife. (2019) 8:e45306. doi: 10.7554/eLife.45306.037

9. Reiter RJ. Pineal melatonin: cell biology of its synthesis and of its physiological interactions. Endocr Rev. (1991) 12:151-180. doi: 10.1210/edrv-12-2-151

10. Fukada Y, Okano T. Circadian clock system in the pineal gland. Mol Neurobiol. (2002) 25:19-30. doi: 10.1385/MN:25:1:019

11. Matsunaga M, Ukena K, Baulieu EE, Tsutsui K. $7 \alpha$-Hydroxypregnenolone acts as a neuronal activator to stimulate locomotor activity of breeding newts by means of the dopaminergic system. Proc Natl Acad Sci USA. (2004) 101:17282-7. doi: 10.1073/pnas.0407176101 
12. Tsutsui K, Inoue K, Miyabara H, Suzuki S, Ogura Y, Haraguchi S. $7 \alpha$-Hydroxypregnenolone mediates melatonin action underlying diurnal locomotor rhythms. J Neurosci. (2008) 28:215867. doi: 10.1523/JNEUROSCI.3562-07.2008

13. Haraguchi S, Koyama T, Hasunuma I, Vaudry H, Tsutsui K. Prolactin increases the synthesis of $7 \alpha$-hydroxypregnenolone, a key factor for induction of locomotor activity, in breeding male newts. Endocrinology. (2010) 151:221122. doi: 10.1210/en.2009-1229

14. Haraguchi S, Koyama T, Hasunuma I, Okuyama S, Ubuka T, Kikuyama S, et al. Acute stress increases the synthesis of $7 \alpha$-hydroxypregnenolone, a new key neurosteroid stimulating locomotor activity, through corticosterone action in newts. Endocrinology. (2012) 153:794-805. doi: 10.1210/en.2011-1422

15. Haraguchi S, Yamamoto Y, Suzuki Y, Hyung Chang J, Koyama T, Sato M, et al. $7 \alpha$-Hydroxypregnenolone, a key neuronal modulator of locomotion, stimulates upstream migration by means of the dopaminergic system in salmon. Sci Rep. (2015) 5:12546. doi: 10.1038/srep12546

16. Fillenz M. Volume transmission in the brain: Novel mechanisms for neuronal transmission. In: Fuxe K, Agnati LF, editors. Advances in Neurosciences, Vol. 1. New York, NY: Raven Press (1991). p. 193-4.

17. Kilic E, Hermann DM, Isenmann S, Bähr M. Effects of pinealectomy and melatonin on the retrograde degeneration of retinal ganglion cells in a novel model of intraorbital optic nerve transection in mice. J Pineal Res. (2002) 32:106-11. doi: 10.1034/j.1600-079x.2002.1823.x

18. Tunç AT, Turgut M, Aslan H, Sahin B, Yurtseven ME, Kaplan S. Neonatal pinealectomy induces Purkinje cell loss in the cerebellum of the chick: a stereological study. Brain Res. (2006) 1067:95-102. doi: 10.1016/j.brainres.2005.10.011

19. Pang Y, Dong J, Thomas P. Characterization, neurosteroid binding and brain distribution of human membrane progesterone receptors $\delta$ and $\varepsilon(\mathrm{mPR} \delta$ and $\mathrm{mPR} \varepsilon$ ) and $\mathrm{mPR} \delta$ involvement in neurosteroid inhibition of apoptosis. Endocrinology. (2013) 154:283-95. doi: 10.1210/en.2012-1772

20. Schumacher M, Mattern C, Ghoumari A, Oudinet JP, Liere P, Labombarda F, et al. Revisiting the roles of progesterone and allopregnanolone in the nervous system: resurgence of the progesterone receptors. Prog Neurobiol. (2014) 113:6-39. doi: 10.1016/j.pneurobio.2013.09.004

21. Belelli D, Lambert JJ. Neurosteroids: endogenous regulators of the GABA receptor. Nat Rev Neurosci. (2005) 6:565-75. doi: 10.1038/nrn1703

22. Bernal J. Thyroid hormone receptors in brain development and function. Nat Clin Pract Endocrinol Metabol. (2007) 3:249-59. doi: 10.1038/ncpendmet0424

23. Falluel-Morel A, Vaudry D, Aubert N, Galas L, Benard M, Basille M, et al. Pituitary adenylate cyclase-activating polypeptide prevents the effects of ceramides on migration, neurite outgrowth, and cytoskeleton remodeling. Proc Natl Acad Sci USA. (2005) 102:2637-42. doi: 10.1073/pnas.04096 81102

24. Koibuchi N, Chin WW. Thyroid hormone action and brain development. Trends Endocrinol Metab. (2000) 11:1238. doi: 10.1016/S1043-2760(00)00238-1
25. Vaudry D, Falluel-Morel A, Leuillet S, Vaudry H, Gonzalez BJ. Regulators of cerebellar granule cell development act through specific signaling pathways. Science. (2003) 300:1532-4. doi: 10.1126/science.1085260

26. Sasahara K, Shikimi H, Haraguchi S, Sakamoto H, Honda S, Harada N, et al. Mode of action and functional significance of estrogen-inducing dendritic growth, Spinogenesis, and synaptogenesis in the developing Purkinje cell. $J$ Neurosci. (2007) 27:7408-17. doi: 10.1523/JNEUROSCI.0710-07.2007

27. Bakkum BW, Benevento LA, Cohen RS. Effects of light/dark- and darkrearing on synaptic morphology in the superior colliculus and visual cortex of the postnatal and adult rat. J Neurosci Res. (1991) 28:6580. doi: 10.1002/jnr.490280107

28. Brooks E, Waters E, Farrington L, Canal MM. Differential hypothalamic tyrosine hydroxylase distribution and activation by light in adult mice reared under different light conditions during the suckling period. Brain Struct Funct. (2011) 216:357-70. doi: 10.1007/s00429-011-0318-9

29. Dulcis D, Spitzer NC. Illumination controls differentiation of dopamine neurons regulating behaviour. Nature. (2008) 456:195-201. doi: 10.1038/nature07569

30. Li Y, Komuro Y, Fahrion JK, Hu T, Ohno N, Fenner KB, et al. Light stimuli control neuronal migration by altering of insulin-like growth factor 1 (IGF-1) signaling. Proc Natl Acad Sci USA. (2012) 109:26305. doi: 10.1073/pnas.1111326109

31. Ohta H, Mitchell AC, McMahon DG. Constant light disrupts the developing mouse biological clock. Pediatr Res. (2006) 60:304-8. doi: 10.1203/01.pdr.0000233114.18403.66

32. Kantermann $\mathrm{T}$, Roenneberg $\mathrm{T}$. Is light-at-night a health risk factor or a health risk predictor? Chronobiol Int. (2009) 26:106974. doi: 10.1080/07420520903223984

33. Wu J, Dauchy RT, Tirrell PC, Wu SS, Lynch DT, Jitawatanarat P, et al. Light at night activates IGF-1R/PDK1 signaling and accelerates tumor growth in human breast cancer xenografts. Cancer Res. (2011) 71:262231. doi: 10.1158/0008-5472.CAN-10-3837

34. Smarr BL, Grant AD, Perez L, Zucker I, Kriegsfeld LJ. Maternal and early-life circadian disruption have long-lasting negative consequences on offspring development and adult behavior in mice. Sci Rep. (2017) 7:3326. doi: 10.1038/s41598-017-03406-4

Conflict of Interest: The authors declare that the research was conducted in the absence of any commercial or financial relationships that could be construed as a potential conflict of interest.

Copyright (C) 2020 Haraguchi and Tsutsui. This is an open-access article distributed under the terms of the Creative Commons Attribution License (CC BY). The use, distribution or reproduction in other forums is permitted, provided the original author(s) and the copyright owner(s) are credited and that the original publication in this journal is cited, in accordance with accepted academic practice. No use, distribution or reproduction is permitted which does not comply with these terms. 